\title{
Thermal Expansion Coefficient of Silicon Nanoparticles with Nanometer Resolution
}

\author{
Bibash Sapkota, Prakash Parajuli, Serdar Ogut and Robert Klie
}

University of Illinois at Chicago, Chicago, Illinois, United States

With the size of electronic devices getting smaller and smaller, it has become important to understand thermal expansion coefficients and thermal properties in sub-nanometer scale [1-3]. However, traditional techniques, such as scanning thermal microscopy or Raman thermometry are limited in spatial resolution due to mechanical constraints or optical diffraction limit [1-3]. In the present work, we will utilize a novel approach of non-contact thermometry based on the combination of low-loss electron energy-loss spectroscopy (EELS) with first principles density functional theory (DFT) modeling to measure the thermal expansion coefficient (TEC) of materials with nano-meter resolution. The approach has been welltested on 2D materials such as TMDs, graphene and $\mathrm{MoS}_{2}$ by $\mathrm{Hu}$ et al [1]. Here, we will extend the approach to $\mathrm{Si}$ nanoparticles.

In the present work, we will study thermal properties of Si nanoparticles with the help of atomic-resolution imaging and electron spectroscopy in an aberration-corrected scanning transmission electron microscope (STEM). We will use the aberration-corrected JEOL-ARM200CF at the University of Illinois at Chicago, equipped with a cold -field emission electron source and a Gatan Continuum GIF. For in-situ heating, we will use Protochips Aduro double-tilt stage on which the sample will be heated up to $1300 \mathrm{~K}$.

According to free electron model, the plasmon energy is related to the temperature of a material through free electron density per unit cell. When changing the temperature, the density of free electrons changes due to expansion in the material which will result in shift in plasmon resonance energy. By combining this experimentally measured shift with theoretical modeling, we can determine thermal expansion coefficient of the material [1]. In this approach, we calculate the plasmon energy of silicon at different lattice parameters by using DFT in the random phase approximation (RPA) within ABINIT [4], which will determine the plasmon energy as a function of lattice parameter.

Figure 1 shows the calculated plasmon-EELS of silicon as a function of lattice parameter. We find a shift of about $0.22 \mathrm{eV}$ in plasmon energy that for a $1 \%$ change in lattice parameter for Si. The plasmon peak for EELS of $\mathrm{Si}$ is fitted by a Lorentzian function to determine the peak position.

Figure 2 shows the experimental data for the plasmon-EELS of silicon at different temperatures. For 300 $\mathrm{K}$ change in temperature, there is the shift of about $0.03 \mathrm{eV}$ in plasmon energy. The effects of particle size, orientation and composition on the TEC, as well as the limits of measuring the local temperature using this approach will be explored in this contribution.[5] 


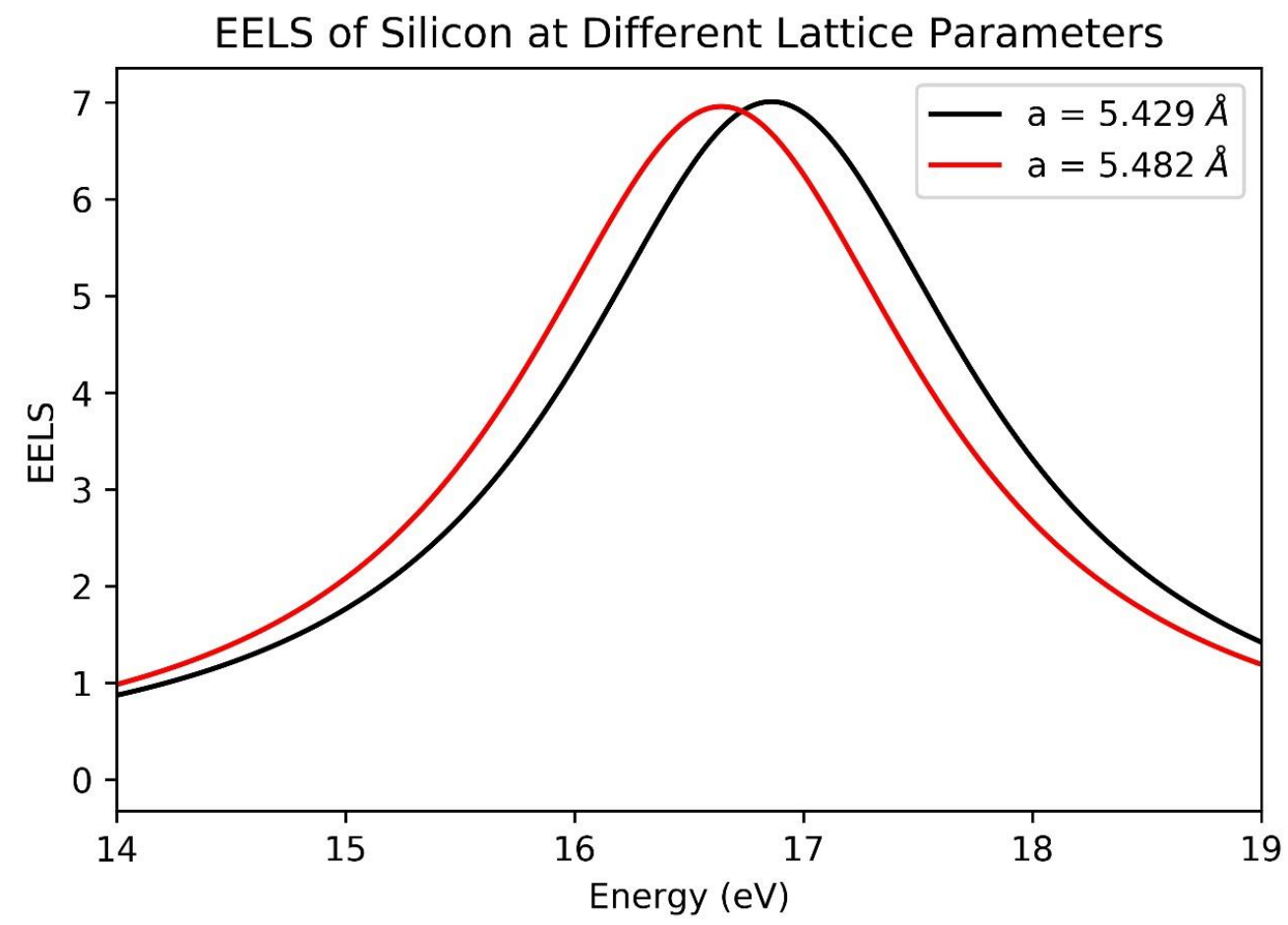

Figure 1. Theoretical low-loss EELS of silicon at different lattice parameters calculated with the help of ABINIT software.

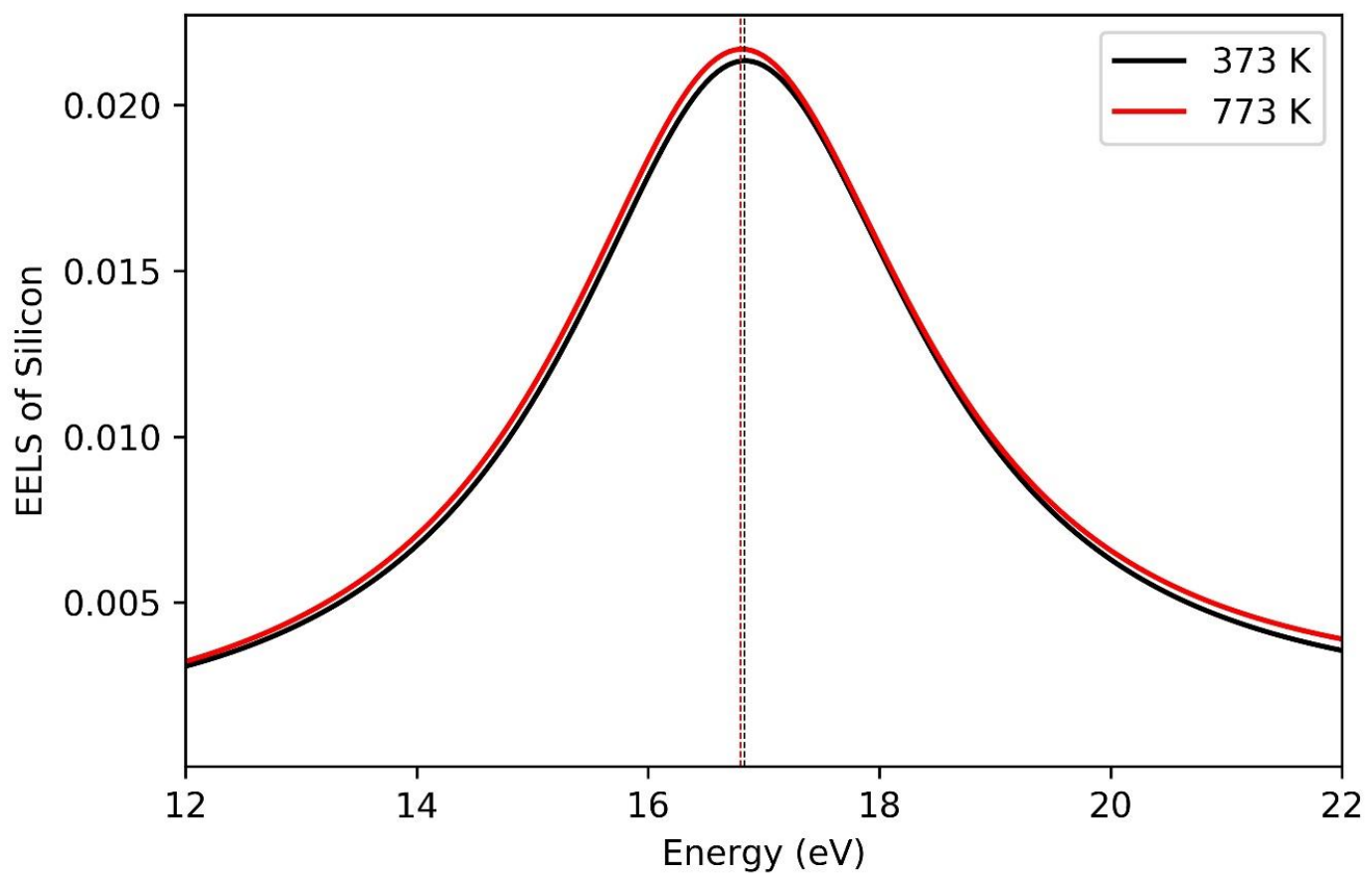

Figure 2. Experimental low-loss EELS of silicon at different temperatures. 


\section{References}

[1] Hu et al., Phys. Rev. Lett. 120, 05590(2018)

[2] Mecklenberg et al., Sci. 347, 6222(2015)

[3] Mecklenberg et al., Phys. Rev. Applied 9, 014005(2018)

[4] Gonze et al., Comput. Phys. Commun. 205, 106-131 (2016)

[5] This work was supported by the National Science Foundation (DMR-18314061). 